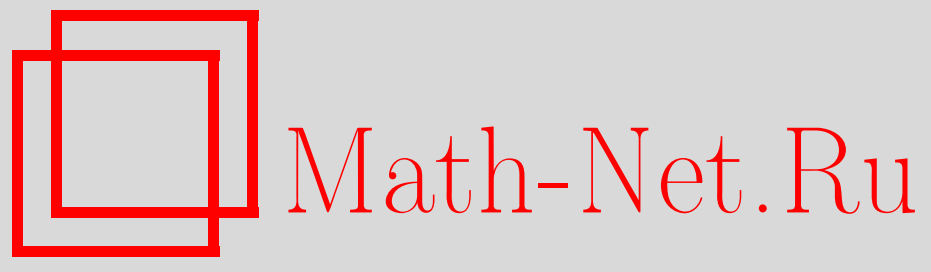

А. А. Беляев, Сингулярные возмущения степеней оператора Лапласа на торе, Матем. заметки, 2013, том 94, выпуск 4, 632-636

DOI: https://doi.org/10.4213/mzm10323

Использование Общероссийского математического портала Math-Net.Ru подразумевает, что вы прочитали и согласны с пользовательским соглашением http://www . mathnet.ru/rus/agreement

Параметры загрузки:

IP : 54.81 .137 .203

26 апреля 2023 г., 11:43:32

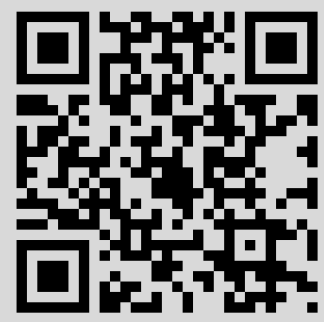




\section{Сингулярные возмущения степеней оператора Лапласа на торе}

\section{А. А. Беляев}

В работе изучаются возмущения степеней оператора Лапласа, заданного на торе. В качестве возмущений выступают сингулярные обобщенные функции, точнее, распределения из пространств мультипликаторов для пространств бесселевых потенциалов.

Основная цель - перенести полученные в [1], [2] для случая $\mathbb{R}^{n}$ теоремы вложения пространств бесселевых потенциалов в пространства мультипликаторов на случай тора $\mathbb{T}^{n}$ и применить абстрактную теорию возмущений для полуограниченных самосопряженных операторов для изучения сингулярных возмущений степеней оператора Лапласа на торе. Мы устанавливаем секториальность и, в случае вещественного потенциала, самосопряженность и ограниченность снизу сингулярно возмущенного оператора (теорема 4). Доказываем равномерную резольвентную сходимость последовательности операторов с потенциалами $q_{m}$ к оператору с потенциалом $q$, когда $q_{m} \rightarrow q$ в пространстве мультипликаторов $M_{\pi}[\alpha,-\alpha]$ (теорема 5). Получаем полноту в $L_{2}\left(\mathbb{T}^{n}\right)$ системы собственных и присоединенных функций возмущенного оператора и находим асимптотику считающей функции его собственных значений (теорема 6). Ранее для случая $n=1$ похожие результаты другим методом были получены в работе [3].

1. Возмущения самосопряженного полуограниченного оператора. Пусть $\mathscr{K}$ гильбертово пространство, а $T: \mathscr{K} \rightarrow \mathscr{K}$ - линейный самосопряженный равномерно положительный оператор. Тогда при $\alpha \in \mathbb{R}$ определены степени $T^{\alpha}$, которые являются также самосопряженными положительными операторами (при $\alpha \geqslant 0$ - равномерно положительными), причем семейство $T^{\alpha}$ обладает полугрупповым свойством. Положим

$$
\mathscr{K}_{\theta} \stackrel{\text { def }}{=} \mathscr{D}\left(T^{\theta / 2}\right) \quad \forall \theta \geqslant 0 .
$$

Заметим, что $T^{\alpha / 2}\left(\mathscr{K}_{\theta}\right)=\mathscr{K}_{\theta-\alpha}$ при $0 \leqslant \alpha \leqslant \theta$, пространство $\mathscr{K}_{\theta}$ является гильбертовым относительно скалярного произведения $\langle x, y\rangle_{\theta}=\left\langle T^{\theta / 2} x, T^{\theta / 2} y\right\rangle_{\mathscr{K}}$ и имеет место непрерывное вложение $\left(\mathscr{K}_{s},\|\cdot\|_{s}\right) \subset\left(\mathscr{K}_{t},\|\cdot\|_{t}\right)$ при $s \geqslant t \geqslant 0$. При $\theta<0$ определим пространство $\mathscr{K}_{\theta}$ как пополнение пространства $\mathscr{K}$ по норме $\|x\|_{\theta}=\left\|T^{\theta / 2} x\right\| \mathscr{K}$. Дуальное скалярное произведение на $\mathscr{K}_{-1} \times \mathscr{K}_{1}$ определим равенством

$$
\langle x, y\rangle_{D}=\left\langle T^{-1 / 2} x, T^{1 / 2} y\right\rangle_{\mathscr{K}} \quad \forall x \in \mathscr{K}_{-1} \quad \forall y \in \mathscr{K}_{1},
$$

где под $T^{-1 / 2}$ понимаем продолжение оператора $T^{-1 / 2}:\left(\mathscr{K},\|\cdot\|_{-1}\right) \rightarrow\left(\mathscr{K}_{1},\|\cdot\|_{\mathscr{K}}\right)$ до изометрии из $\mathscr{K}_{-1}$ на $\mathscr{K}$.

Назовем оператор $Q: \mathscr{K}_{1} \rightarrow \mathscr{K}_{-1}$ симметричным, если вещественна форма $\langle Q(v), v\rangle_{D}$. Если область определения $\mathscr{D}(Q)$ плотна в $\mathscr{K}_{1}$, то с помощью дуального скалярного произведения определен сопряженный оператор $Q^{*}: \mathscr{K}_{1} \rightarrow \mathscr{K}_{-1}$. Определенный на всем пространстве $\mathscr{K}_{1}$ симметрический оператор оказывается самосопряженным.

Продолжим изометрический оператор $T:\left(\mathscr{K}_{2},\|\cdot\|_{1}\right) \rightarrow\left(\mathscr{K},\|\cdot\|_{-1}\right)$ до всюду определенного изометрического оператора $\mathbf{T}: \mathscr{K}_{1} \rightarrow \mathscr{K}_{-1}$. Тогда

$$
\begin{array}{lll}
\langle\mathbf{T} u, v\rangle_{D}=\left\langle T^{1 / 2} u, T^{1 / 2} v\right\rangle_{\mathscr{K}} & & \forall u, v \in \mathscr{K}_{1}, \\
\langle\mathbf{T} u, u\rangle_{D}=\left(\|u\|_{1}\right)^{2}>0 & & \forall u \neq 0, u \in \mathscr{K}_{1},
\end{array}
$$

Работа выполнена при поддержке Российского фонда фундаментальных исследований (грант № 13-01-0705).

DOI: $10.4213 / \mathrm{mzm} 10323$ 
и оператор $\mathbf{T}$ самосопряжен. Приведем в удобном для дальнейшего применения модификации теорем о возмущениях самосопряженного оператора Т (см. теоремы VI.1.33, VI.2.1 и VI.2.6 из монографии [4]).

Утверждение 1. Пусть линейный оператор $Q: \mathscr{K}_{1} \rightarrow \mathscr{K}_{-1}$ определен на всем пространстве $\mathscr{K}_{1}$ и подчинен оператору $\mathbf{T}: \mathscr{K}_{1} \rightarrow \mathscr{K}_{-1}$ в смысле форм с $\mathbf{T}$-гранью $\alpha<1$, m.e.

$$
\left|\langle Q v, v\rangle_{D}\right| \leqslant \alpha \cdot\langle\mathbf{T} v, v\rangle_{D}+\beta \cdot\left(\|v\|_{\mathscr{K}}\right)^{2} \quad \forall v \in \mathscr{K}_{1}
$$

при некотором $\beta>0$. Тогда квадратичная форма

$$
(t+q)(v) \stackrel{\text { def }}{=}\langle(\mathbf{T}+Q) v, v\rangle_{D}
$$

с областью определения $\mathscr{D}(t+q)=\mathscr{K}_{1}$ замкнута и секториальна в $\left(\mathscr{K},\|\cdot\|_{\mathscr{K}}\right)$. Если оператор $Q, \kappa$ тому же, симметричен, то квадратичная форма $t+q$ полуограничена снизу относительно нормы $\|\cdot\|_{\mathscr{K}}$.

УтвеРЖДЕнИЕ 2. Пусть линейный оператор $Q: \mathscr{K}_{1} \rightarrow \mathscr{K}_{-1}$ определен на всем $\mathscr{K}_{1}$ u подчинен оператору $\mathbf{T}$ в смысле форм с $\mathbf{T}$-гранъю $\alpha<1$. Тогда сужение $\mathbf{T} \widetilde{+} Q$ оператора $\mathbf{T}+Q$ на пространство $\mathscr{D}(\mathbf{T} \widetilde{+} Q)=\left\{v \in \mathscr{K}_{1} \mid(\mathbf{T}+Q) v \in \mathscr{K}\right\}$ является секториальным оператором в $\mathscr{K}$. Если при этом $Q$ симметричен, то $\mathbf{T} \widetilde{+} Q$ самосопряжен в $(\mathscr{K},\langle\cdot, \cdot\rangle \mathscr{K})$.

Вопрос о равномерной резольвентной сходимости операторов $\mathbf{T} \widetilde{+} Q_{n}$ к оператору $\mathbf{T} \widetilde{+} Q$ исследован в [1] и [2], где, в частности, доказано следующее

Утверждение 3. Пусть $Q: \mathscr{K}_{1} \rightarrow \mathscr{K}_{-1}$ u $Q_{m}: \mathscr{K}_{1} \rightarrow \mathscr{K}_{-1}$ - всюду определенные на $\mathscr{K}_{1}$ линейные операторы, $Q$ - T-подчинен с $\mathbf{T}$-гранъю $\alpha<1$ u $Q_{m} \stackrel{m \rightarrow \infty}{\longrightarrow} Q$ по норме пространства $\mathscr{B}\left(\mathscr{K}_{1}, \mathscr{K}_{-1}\right)$ ограниченных операторов из $\mathscr{K}_{1}$ в $\mathscr{K}_{-1}$. Тогда имеет место равномерная резольвентная сходимость

$$
\mathbf{T} \tilde{+} Q_{m} \stackrel{R}{\Longrightarrow} \mathbf{T} \tilde{+} Q .
$$

2. Оператор Лапласа и пространства мультипликаторов на торе. Пусть $S^{1}$ единичная окружность, $\mathbb{T}^{n}=\left\{\left(z_{1}, \ldots, z_{n}\right) \mid z_{j} \in S^{1} \forall j=1, \ldots, n\right\}-n$-мерный тор и $\Delta-$ оператор Лапласа на $\mathbb{T}^{n}$ с областью определения $\mathscr{D}(\Delta)=C^{2}\left(\mathbb{T}^{n}\right) \subset L^{2}\left(\mathbb{T}^{n}\right)$. Известно, что оператор $-\Delta$ симметричен и неотрицателен в $L^{2}\left(\mathbb{T}^{n}\right)$. Обозначим через $D\left(\mathbb{T}^{n}\right)$ пространство бесконечно дифференцируемых функций на $\mathbb{T}^{n}$. Функцию $f \in D\left(\mathbb{T}^{n}\right)$ естественным образом отождествим с единственной функцией $\Pi(f) \in C_{\pi}^{\infty}\left(\mathbb{R}^{n}\right)$, где $C_{\pi}^{\infty}\left(\mathbb{R}^{n}\right)$ - пространство бесконечно гладких $2 \pi$-периодических функций на $\mathbb{R}^{n}$. Функции

$$
f_{k}=\Pi^{-1}\left(g_{k}\right) \in D\left(\mathbb{T}^{n}\right), \quad k \in \mathbb{Z}^{n}, \quad \text { где } \quad g_{k}(x)=(2 \pi)^{-n / 2} e^{-i\langle k, x\rangle} \quad \forall x \in \mathbb{R}^{n},
$$

образуют в $L_{2}\left(\mathbb{T}^{n}\right)$ ортонормированный базис собственных функций оператора $\Delta$ с собственными числами $\lambda_{k}=-|k|^{2}$.

Дадим определение пространства бесселевых потенциалов на торе, которое будем обозначать через $H_{p}^{\alpha}\left(\mathbb{T}^{n}\right)$. Обозначим через $P_{\alpha}$ оператор умножения на функцию $p_{\alpha}(y)=$ $\left(1+|y|^{2}\right)^{\alpha / 2}$, а через $\mathscr{F}$ и $\mathscr{F}^{-1}-$ прямое и обратное преобразование Фурье. Рассмотрим оператор

$$
J_{\alpha}=\mathscr{F}^{-1} \circ P_{\alpha} \circ \mathscr{F}: S^{\prime}\left(\mathbb{R}^{n}\right) \rightarrow S^{\prime}\left(\mathbb{R}^{n}\right),
$$

где $S^{\prime}\left(\mathbb{R}^{n}\right)$ - пространство умеренно растущих распределений (на котором корректно определено преобразование Фурье). Несложно показать, что оператор $J_{\alpha}$ отображает пространство $C_{\pi}^{\infty}\left(\mathbb{R}^{n}\right)$ на себя. Поэтому корректно определен оператор

$$
J_{\alpha, \pi} \stackrel{\text { def }}{=} \Pi^{-1} \circ J_{\alpha} \circ \Pi: D\left(\mathbb{T}^{n}\right) \rightarrow D\left(\mathbb{T}^{n}\right) .
$$

Этот оператор можно естественно продолжить на пространство распределений $D^{\prime}\left(\mathbb{T}^{n}\right)$, т.е. пространство линейных непрерывных функционалов на $D\left(\mathbb{T}^{n}\right)$. Такое продолжение 
реализуется формулой $J_{\alpha, \pi}(u)(f) \stackrel{\text { def }}{=} u\left(J_{\alpha, \pi}(f)\right)$, где $u$ - произвольный фиксированный элемент из $D^{\prime}\left(\mathbb{T}^{n}\right)$, а $f$ пробегает все пространство $D\left(\mathbb{T}^{n}\right)$.

Пространство $L_{1}\left(\mathbb{T}^{n}\right)$ естественным образом вложено в $D^{\prime}\left(\mathbb{T}^{n}\right)$, при этом функции $f \in$ $L_{1}\left(\mathbb{T}^{n}\right)$ порождают регулярные функционалы, для которых мы будем сохранять обозначение $f$. Теперь при $p \geqslant 1, \alpha \in \mathbb{R}$ положим

$$
H_{p}^{\alpha}\left(\mathbb{T}^{n}\right) \stackrel{\text { def }}{=}\left\{u \in D^{\prime}\left(\mathbb{T}^{n}\right) \mid J_{\alpha, \pi}(u) \in L_{p}\left(\mathbb{T}^{n}\right)\right\} \quad \text { и } \quad\|f\|_{H_{p}^{\alpha}\left(\mathbb{T}^{n}\right)} \stackrel{\text { def }}{=}\left\|J_{\alpha, \pi}(f)\right\|_{L_{p}\left(\mathbb{T}^{n}\right)} .
$$

Из определения следует, что $H_{p}^{0}\left(\mathbb{T}^{n}\right)=L_{p}\left(\mathbb{T}^{n}\right)$. Отметим, что пространства $H_{p}^{\alpha}\left(\mathbb{T}^{n}\right)$ можно определить по-другому (см. [5; пункт 4.11.1]). Для этого используется тот факт, что функции

$$
g_{k}(x)=(2 \pi)^{-n / 2} e^{-i\langle k, x\rangle}, \quad k \in \mathbb{Z}^{n},
$$

образуют базис в пространстве $C_{\pi}^{\infty}\left(\mathbb{R}^{n}\right)$ и $c_{k}\left(J_{\alpha, \pi}(f)\right)=\left(1+|k|^{2}\right)^{\alpha / 2} c_{k}(f)$, где через $c_{k}(g)$ обозначены коэффициенты Фурье функции $g$. Отметим также, что при целых $m \geqslant 0$ пространство $H_{2}^{m}\left(\mathbb{T}^{n}\right)$ совпадает с пространством Соболева $W_{2}^{m}\left(\mathbb{T}^{n}\right)$, причем их нормы эквивалентны.

Поскольку при $\alpha \geqslant 0$ сужение оператора $J_{\alpha, \pi}$ на $H_{2}^{\alpha}\left(\mathbb{T}^{n}\right)$ является самосопряженным оператором в $L_{2}\left(\mathbb{R}^{n}\right)$ и $(I-\Delta) f=J_{2, \pi} f, f \in D\left(\mathbb{T}^{n}\right)$, где $I$ - тождественный оператор, то оператор $L_{0}=-\Delta$ в $L_{2}\left(\mathbb{T}^{n}\right)$ с областью определения $C^{2}\left(\mathbb{T}^{n}\right)$ имеет самосопряженное замыкание $L$, область определения которого $\mathscr{D}(L)$ совпадает с $H_{2}^{2}\left(\mathbb{T}^{n}\right) \subset L_{2}\left(\mathbb{T}^{n}\right)$. Далее под $-\Delta$ мы будем понимать именно оператор $L$.

Определим при $\alpha \geqslant 0$ оператор $(-\Delta)^{\alpha}$ с областью определения $H_{2}^{2 \alpha}\left(\mathbb{T}^{n}\right)$, полагая

$$
(-\Delta)^{\alpha}(f) \stackrel{\text { def }}{=} \sum_{k \in \mathbb{Z}^{n}}|k|^{2 \alpha} c_{k}(f) f_{k}, \quad f \in H_{2}^{2 \alpha}\left(\mathbb{T}^{n}\right),
$$

где $c_{k}(f)$ - коэффициенты Фурье функции $f$.

УтвеРЖДЕнИЕ 4. При $\alpha \geqslant 0$ оператор $T_{\alpha}=I+(-\Delta)^{\alpha}$ с областью определения $\mathscr{D}\left(T_{\alpha}\right)=$ $H_{2}^{2 \alpha}\left(\mathbb{T}^{n}\right)$ самосопряжен и равномерно положителен в $L_{2}\left(\mathbb{T}^{n}\right)$.

Следовательно, в соответствии с изложенным в п. 1 для оператора $T_{\alpha}$ определена шкала пространств $\mathscr{K}_{\theta, \alpha}=\mathscr{D}\left(\left(T_{\alpha}\right)^{\theta / 2}\right)$.

Теорема 1. Пусть $\alpha>0$. Тогда для любого $\theta \geqslant 0$ пространства $\mathscr{K}_{\theta, \alpha}$ u $H_{2}^{\theta \alpha}\left(\mathbb{T}^{n}\right)$ совпадают и их нормы эквивалентны.

ДокАЗАТЕЛьСтво основано на оценках норм через коэффициенты Фурье.

Пусть $r \in(0, \pi), B_{r}(x)=\left\{y \in \mathbb{R}^{n}|| y-x \mid<r\right\}$,

$$
\mathscr{U}=\left\{U_{j}=\pi\left(B_{r}\left(x_{j}\right)\right) \mid x_{j} \in \mathbb{R}^{n}, j=1, \ldots, N\right\}
$$

- конечное открытое покрытие тора $\mathbb{T}^{n}$ (здесь $\pi$ - естественное отображение $\mathbb{R}^{n}$ на тор $\mathbb{T}^{n}$ ) и $\psi=\left\{\psi_{j} \mid j=1, \ldots, N\right\}$ - подчиненное этому покрытию гладкое разбиение единицы. Для $f \in D\left(U_{j}\right)$ положим

$$
f_{U_{j}}(x)=(\Pi(f))(x) \quad \text { при } \quad x \in B_{r}\left(x_{j}\right) \quad \text { и } \quad f_{U_{j}}(x)=0 \quad \text { при } \quad x \in \mathbb{R}^{n} \backslash B_{r}\left(x_{j}\right) .
$$

Введем в $H_{p}^{\alpha}\left(\mathbb{T}^{n}\right)$ новую норму

$$
\|f\|_{\alpha, p, \psi} \stackrel{\text { def }}{=} \sum_{j=1, \ldots, N}\left\|\left(\psi_{j} f\right)_{U_{j}}\right\|_{H_{p}^{\alpha}\left(\mathbb{R}^{n}\right)} \quad \forall f \in H_{p}^{\alpha}\left(\mathbb{T}^{n}\right) .
$$

Теорема 2. Нормы $\|\cdot\|_{\alpha, p, \psi} u\|\cdot\|_{H_{p}^{\alpha}\left(\mathbb{T}^{n}\right)}$ эквивалентны на $H_{p}^{\alpha}\left(\mathbb{T}^{n}\right)$. 
Доказательство использует эквивалентные нормы на $H_{p}^{\alpha}\left(\mathbb{R}^{n}\right)$, вводимые с помощью обратного преобразования Фурье функции $\left(1+|y|^{2}\right)^{\alpha / 2}$ (см. [6], [7]).

Далее норму в пространстве $H_{2}^{\alpha}\left(\mathbb{T}^{n}\right)$ для краткости будем обозначать через $\|\cdot\|_{\alpha}$. Распределение $q \in D^{\prime}\left(\mathbb{T}^{n}\right)$ назовем мультипликатором из пространства $H_{2}^{k}\left(\mathbb{T}^{n}\right)$ в $H_{2}^{-l}\left(\mathbb{T}^{n}\right)$ (предполагаем, что $k$ и $l$ неотрицательны), если найдется постоянная $C>0$ такая, что для всех $f \in D\left(\mathbb{T}^{n}\right)$ выполняется оценка $\|f q\|_{-l} \leqslant C\|f\|_{k}$. В этом случае оператор умножения $M_{q} f=f q$ продолжается по непрерывности как ограниченный оператор из пространства $H_{2}^{k}\left(\mathbb{T}^{n}\right)$ в пространство $H_{2}^{-l}\left(\mathbb{T}^{n}\right)$. Множество таких мультипликаторов образуют линейное нормированное пространство, если в качестве нормы элемента $q$ взять норму ограниченного оператора $M_{q}$. Пространство таких мультипликаторов обозначим через $M[k,-l]$. Справедлива важная

Лемма 1. 1) При $k>n / 2,0 \leqslant l \leqslant k$ верна мультипликативная оценка

$$
\|f g\|_{l} \leqslant C_{k, l}\|f\|_{k}\|g\|_{l} \quad \forall f, g \in D\left(\mathbb{T}^{n}\right) .
$$

2) При $0 \leqslant l \leqslant k \leqslant n / 2$, при любом $\varepsilon>0$ верна мультипликативная оценка

$$
\|f g\|_{l-\varepsilon} \leqslant C_{k, l, \varepsilon}\|f\|_{k}\|g\|_{l} \quad \forall f, g \in D\left(\mathbb{T}^{n}\right) .
$$

ДокАЗАТЕЛьство использует теорему 2 и аналогичные оценки в $\mathbb{R}^{n}$ из [2].

Теорема 3. а) При $k>n / 2,0 \leqslant l \leqslant k$ справедливо равенство

$$
H_{2}^{-l}\left(\mathbb{T}^{n}\right)=M[k,-l]_{\pi}
$$

и нормы соответствующих пространств эквивалентны.

b) При $k \leqslant n / 2,0 \leqslant l \leqslant k$ при любом $\varepsilon>0$ справедливо непрерывное вложение

$$
H_{n / k}^{-l+\varepsilon}\left(\mathbb{T}^{n}\right) \subset M[k,-l]_{\pi} .
$$

Доказательство опирается на лемму 1 и тот факт, что $1 \in H_{2}^{k}\left(\mathbb{T}^{n}\right)$ и, следовательно,

$$
M_{u}(1)=u \in H_{2}^{-l}\left(\mathbb{T}^{n}\right) \quad \forall u \in M[k,-l]_{\pi} .
$$

3. Основные теоремы. Далее $\mathscr{K}=L_{2}\left(\mathbb{T}^{n}\right), \mathscr{K}_{\theta}=\mathscr{K}_{\theta, \alpha}=H_{2}^{\theta \alpha}\left(\mathbb{T}^{n}\right)$ при $\theta \geqslant 0$ (в частности, $\mathscr{K}_{1}=H_{2}^{\alpha}\left(\mathbb{T}^{n}\right)$ и $\left.\mathscr{K}_{-1}=H_{2}^{-\alpha}\left(\mathbb{T}^{n}\right)\right), T_{\alpha}=I+(-\Delta)^{\alpha}: \mathscr{K} \rightarrow \mathscr{K}_{\text {и }} \mathbf{T}=\mathbf{T}_{\alpha}: \mathscr{K}_{1} \rightarrow \mathscr{K}_{-1}-$ оператор, соответствующий оператору $T_{\alpha}$.

Доказательства теорем 4 и 5 опираются на теорему 3 и применение абстрактных утверждений 1-3 в описанной выше ситуации.

Tеорема 4. Пусть $q \in M[\alpha,-\alpha]_{\pi}$ и оператор $Q=M_{q}: H_{2}^{\alpha}\left(\mathbb{T}^{n}\right) \rightarrow H_{2}^{-\alpha}\left(\mathbb{T}^{n}\right)$ подчинен оператору $\mathbf{T}$ в смысле форм с $\mathbf{T}$-гранъю $b<1$. Тогда оператор

$$
S=(-\Delta)^{\alpha}+Q: H_{2}^{\alpha}\left(\mathbb{T}^{n}\right) \rightarrow H_{2}^{-\alpha}\left(\mathbb{T}^{n}\right)
$$

определен на $H_{2}^{\alpha}\left(\mathbb{T}^{n}\right)$, а его сужение $L=(-\Delta)^{\alpha} \widetilde{+} Q$ на

$$
\mathscr{D}(L)=\left\{u \in H_{2}^{\alpha}\left(\mathbb{T}^{n}\right) \mid S(u) \in L_{2}\left(\mathbb{T}^{n}\right)\right\}
$$

является секториальным оператором в $L_{2}\left(\mathbb{T}^{n}\right)$. Если $Q$ симметричен, то L самосопряжен и полуограничен снизу в $L_{2}\left(\mathbb{T}^{n}\right)$.

СлеДСтвие 1. Пусть $\alpha>n / 2, q \in H_{2}^{-\alpha}\left(\mathbb{T}^{n}\right)$ или $0<\alpha \leqslant n / 2, \varepsilon>0 u q \in H_{n / \alpha}^{-\alpha+\varepsilon}\left(\mathbb{T}^{n}\right)$. Тогда $q \in M[\alpha,-\alpha]_{\pi}$ и верно утверждение теоремы 4. 
Теорема 5. Пусть выполнены условия теоремы 4 и последовательность $q_{m}$ сходится $\kappa q$ по норме пространства $M[\alpha,-\alpha]_{\pi}$. Тогда найдется $m_{0} \in \mathbb{N}$ такое, что при $m \geqslant m_{0}$ корректно определены оператори

$$
L_{m}=(-\Delta)^{\alpha} \widetilde{+} Q_{m}, \quad \text { где } \quad Q_{m}=M_{q_{m}}: H_{2}^{\alpha}\left(\mathbb{T}^{n}\right) \rightarrow H_{2}^{-\alpha}\left(\mathbb{T}^{n}\right),
$$

и выполнено

$$
L_{m} \stackrel{R}{\Longrightarrow} L \quad \text { npu } \quad m \rightarrow \infty .
$$

Пусть $N(r, A)$ - сумма кратностей всех собственных значений $\lambda \in D_{r}=\{z \in \mathbb{C}|| z \mid<$ $r\} \subset \mathbb{C}$ оператора $A$.

Teopema 6. Пусть $q \in M[\alpha,-\alpha]_{\pi}$ u onepamop

$$
Q=M_{q}: H_{2}^{\alpha}\left(\mathbb{T}^{n}\right) \rightarrow H_{2}^{-\alpha}\left(\mathbb{T}^{n}\right)
$$

компактен. Тогда

1) оператор

$$
L=(-\Delta)^{\alpha} \widetilde{+} Q: L_{2}\left(\mathbb{T}^{n}\right) \rightarrow L_{2}\left(\mathbb{T}^{n}\right)
$$

имеет компактную резольвенту;

2) система его собственных и присоединенных функиий полна в $\mathscr{K}=L_{2}\left(\mathbb{T}^{n}\right)$;

3) при $r \rightarrow \infty$ справедливо соотношение

$$
N(r, L) \sim N\left(r,(-\Delta)^{\alpha}\right) \sim C_{\alpha} r^{n /(2 \alpha)} .
$$

ДокАзАтельство использует теоремы Келдыша-Маркуса-Мацаева [8; теоремы 4.2, $4.3,8.2]$.

СледСтвиЕ 2. Пусть выполнены условия следствия теоремы 4. Тогда справедливо утверждение теоремы 6.

Автор выражает глубокую благодарность проф. А. А. Шкаликову за постановку задач и внимание к работе.

\section{СПИСОК ЦИТИРОВАННОЙ ЛИТЕРАТУРЫ}

[1] М.И. Нейман-Заде, А.А. Шкаликов, Матем. заметки, 66:5 (1999), 723-733. [2] M. I. Neiman-Zade, A. A. Shkalikov, Russian J. Math. Phys., 13:1 (2006), 70-78. [3] В. А. Михайлец, В. Н. Молибога, Матем. заметки, 91:4 (2012), 629-632. [4] Т. Като, Теория возмущений линейных операторов, Мир, М., 1972. [5] Х. Трибель, Теория интерполяиии, функииональные пространства, дифференииальные операторы, Мир, М., 1980. [6] О. В. Бесов, Тр. МИАН СССР, 60, Изд-во АН СССР, М., 1961, 42-81. [7] R. S. Strichartz, J. Math. Mech., 16 (1967), 1031-1060. [8] А. С. Маркус, Введение в спектральную теорию полиномиальных операторных пучков, Штиинца, Кишинев, 1986.

\section{А. А. Беляев}

Поступило

Московский государственный 